\title{
Comparison between the degree of motor unit short-term synchronization and recurrence quantification analysis of the surface EMG in two human muscles
}

\author{
J.L. Dideriksen, D. Falla, M. Bækgaard, M.L. Mogensen, K.L. Steimle, D. Farina* \\ Center for Sensory-Motor Interaction (SMI), Department of Health Science and Technology, Aalborg University, Aalborg, Denmark
}

\section{A R T I C L E I N F O}

\section{Article history:}

Accepted 17 September 2009

Available online 13 October 2009

\section{Keywords:}

Motor unit

Recurrence quantification analysis

Surface EMG

Synchronization

\begin{abstract}
A B S T R A C T
Objective: To verify if non-linear recurrence analysis of the surface EMG is a suitable tool for assessing motor unit short-term synchronization.

Methods: Surface and intramuscular EMG signals were recorded from the abductor digiti minimi and vastus medialis muscles of 12 and 10 healthy men, respectively, during isometric contractions. In the abductor digiti minimi, EMG signals were additionally recorded after a contraction sustained for $1 \mathrm{~min}$ at $50 \%$ of the maximal force. In both muscles, percent of determinism (\%DET) was estimated from the surface EMG and common input strength (CIS) index was computed from motor unit recordings.

Results: For both muscles, CIS did not correlate with \%DET (abductor digiti minimi: $R^{2}=0.11, P=0.12$; vastus medialis: $R^{2}=0.04, P=0.56$ ). Although the values of CIS for the vastus medialis were lower than those of the abductor digiti minimi $(P<0.001)$, the \%DET values did not differ between the two muscles (71.6 $\pm 5.5 \%$ vs $66.9 \pm 8.7 \% ; P=0.12$ ).

Conclusion: The variable \%DET extracted from the surface EMG is a poor indicator of the degree of motor unit short-term synchronization.

Significance: The study provides a systematic evaluation of a technique previously proposed for the estimation of a clinically relevant characteristic of motor unit behavior.

(c) 2009 International Federation of Clinical Neurophysiology. Published by Elsevier Ireland Ltd. All rights
\end{abstract}

reserved.

\section{Introduction}

The discharges of motor units often show a small, above-chance probability of occurring in synchrony (Buchthal and Madsen, 1950; Sears and Stagg, 1976). This observation reflects common pre-synaptic input to the motor neurons from branched neurons in the spinal cord. The phenomenon is known as motor unit short-term synchronization and its measure provides information on the function of last-order neurons with divergent projections to motor neurons (Nordstrom et al., 1992; Farmer et al., 1993; Semmler, 2002). Abnormal levels of motor unit short-term synchronization have been shown to be related to pathological conditions, such as Klippel-Feil syndrome (Semmler, 2002).

A method to quantify motor unit short-term synchronization involves computing cross-histograms between pairs of motor unit spike trains (Nordstrom et al., 1992). If the discharges are synchronized above a level due to chance, the cross-histogram shows a significant peak, and the area provides quantification of the strength of short-term synchronization. One of the most common indices

\footnotetext{
* Corresponding author. Address: Center for Sensory-Motor Interaction (SMI), Department of Health Science and Technology, Aalborg University, Fredrik Bajers Vej 7 D-3, DK-9220 Aalborg, Denmark. Tel.: +45 99408821; fax: +45 98154008.

E-mail address: df@hst.aau.dk (D. Farina).
}

extracted from cross-histograms of motor unit pairs is the common input strength (CIS) (Nordstrom et al., 1992), which is computed as the area of the cross-histogram peak above the level due to chance, divided by the duration of the recording.

The computation of cross-histograms for quantifying synchronization requires lengthy recordings, a sufficient number of identified motor units, and precise intramuscular EMG signal decomposition (Semmler and Nordstrom, 1999). These requirements and the invasiveness of the method limit the measure of motor unit short-term synchronization to laboratory conditions. Because of the difficulties in applying this approach, non-invasive methods, i.e. based on the surface EMG, have also been explored for estimating the strength of motor unit short-term synchronization (Milner-Brown et al., 1973, 1975; Del Santo et al., 2006).

Among the methods used to process the interference EMG signal, the percentage of determinism (\%DET), extracted from recurrence quantification analysis (Webber et al., 1995), describes the number of recurrent structures in the signal, which may be associated to the degree of motor unit synchronization (Filligoi and Felici, 1999). Because of the potential advantages of using \%DET of the surface EMG for quantifying motor unit synchronization, there have been attempts to provide direct evidence for this association (Del Santo et al., 2006, 2007). For example, the increase in \%DET observed during fatiguing contractions has been partly attributed to an increase in 
the degree of motor unit synchronization (Felici et al., 2001). However, simulation analysis demonstrated that the type of information provided by \%DET is highly correlated to that obtained by spectral analysis of the surface EMG (Farina et al., 2002a). Therefore, it is expected that \%DET is affected by several factors, as is spectral EMG analysis (Farina et al., 2004), which would mask its association to one specific physiological mechanism.

Despite the problems outlined by simulation studies (Farina et al., 2002a), a high correlation has been identified between \%DET and the magnitude of the synchronization peak area of the crosshistogram estimated from concurrent recordings of intramuscular and surface EMG signals (Del Santo et al., 2007). However, in these studies the degree of synchronization was pharmacologically changed which allowed a rather large range of synchronization values to be investigated (Del Santo et al., 2006). It is not known if the results observed in these conditions are also valid in physiological conditions and thus if \%DET of the surface EMG can be used as an alternative for the direct estimation of the strength of motor unit shortterm synchronization.

This study addresses the association between \%DET and a direct measure of synchronization by concurrent recordings of intramuscular and surface EMG signals from two muscles. The correlation between the direct measure of synchronization provided by pairs of motor units and the \%DET was determined across subjects within the same muscle and compared across muscles. The study was conducted using measurements from the abductor digiti minimi and vastus medialis muscles since it was expected that these two muscles would present significantly different degrees of motor unit short-term synchronization (Datta et al., 1991; De Luca et al., 1993; Kim et al., 2001). The aim of the study was to verify if recurrence quantification analysis is an applicable tool for assessing motor unit short-term synchronization in physiological conditions.

\section{Materials and methods}

\subsection{Subjects}

Two groups of subjects participated in two experimental sessions after providing written informed consent. The first group consisted of 12 healthy men (age, mean \pm SD, $25.0 \pm 3.3$ yrs; weight: $82.2 \pm 12.6 \mathrm{~kg}$; height: $181.5 \pm 4.6 \mathrm{~cm}$ ) and the second group of 10 healthy men (age, $26.2 \pm 4.2$ yrs; weight: $84.3 \pm 13.7 \mathrm{~kg}$; height: $182.2 \pm 5.6 \mathrm{~cm}$ ). All subjects were right-hand dominant and free from pain in the upper and lower limbs. Ethical approval for the study was granted by the local Ethics Committee (N-20070019) and all procedures were conducted according to the Declaration of Helsinki.

\subsection{Surface EMG recording}

Surface EMG signals were detected from the abductor digiti minimi muscle of the right hand in the first group of subjects and from the vastus medialis muscle of the right thigh in the second group. Recordings from the abductor digiti minimi were performed with a linear array of 16 electrodes ( $2.5 \mathrm{~mm}$ interelectrode distance; LISiN-OT Bioelettronica, Torino, Italy) whereas an array with eight electrodes ( $5 \mathrm{~mm}$ interelectrode distance) was used for the vastus medialis muscle. In both experiments, the position of the electrode array was determined by varying the location of the array during moderate contractions, until propagation of action potentials was detectable on at least four channels (visual inspection). The subject's skin was prepared by gentle local abrasion using abrasive paste (Medic-Every, Parma, Italy), cleaned with water and the electrode secured with adhesive tape.
Surface EMG signals were amplified (128-channel EMG amplifier, LISiN-OT Bioelettronica, Torino, Italy; $-3 \mathrm{~dB}$ bandwidth $10-$ $500 \mathrm{~Hz}$ ) with a gain of 5000 , sampled at $2048 \mathrm{~Hz}$, and converted to digital form by a 12-bit A/D converter. A ground electrode was placed around the right wrist.

\subsection{Intramuscular EMG recordings}

In both experiments, action potentials from single motor units were recorded with two pairs of Teflon-coated stainless steel wires (diameter: $0.1 \mathrm{~mm}$; A-M Systems, Carlsborg, WA) inserted at two locations in the abductor digiti minimi or the vastus medialis muscle via 25 -gauge disposable hypodermic needles. The wires were cut to expose only the cross section and the two pairs of electrodes were inserted at a distance of $1-1.5 \mathrm{~cm}$ in the direction transverse to the muscle fibers and $\sim 1 \mathrm{~cm}$ proximal to the surface array.

The bipolar intramuscular EMG signals were amplified (Counterpoint EMG, DANTEC Medical, Skovlunde, Denmark), band-pass filtered $(500 \mathrm{~Hz}-5 \mathrm{kHz})$, sampled at $10,000 \mathrm{~Hz}$, and stored after 12-bit A/D conversion. Intramuscular signals were displayed on a computer screen to provide visual feedback to the subject.

\subsection{Force recordings}

For the measures on the abductor digiti minimi, the subjects' right forearm was secured in a mould with the wrist joint in a neutral position and the palm facing upwards. The hand and the first four fingers were secured in a rigid piece and the fifth finger was free to move (Fig. 1A). A force transducer (Interface MB-100, USA) was aligned with the distal interphalangeal joint of the fifth finger in order to measure abduction force of the fifth finger. The distance between the fifth finger and the force transducer when the hand was in a relaxed position was $\sim 1 \mathrm{~mm}$.

For the measures on the vastus medialis, the subject was seated comfortably on a chair with the hip and knee in $90^{\circ}$ of flexion, the thigh firmly secured by a metal bar mounted on the chair and the chest secured to the chair by a belt. A strap which was connected to a force transducer (Interface MB-100, USA) was fastened around the right ankle to measure knee extension force (Fig. 1B).

Force signals in both experiments were amplified (Miso II, LI$\mathrm{SiN-OT} \mathrm{Bioelettronica,} \mathrm{Torino,} \mathrm{Italy)} \mathrm{and} \mathrm{their} \mathrm{output} \mathrm{was} \mathrm{displayed}$ on an oscilloscope as visual feedback to the subject.

\subsection{Procedures}

In both experiments, subjects performed three maximum voluntary isometric contractions (MVC) of 3-4 s duration. Verbal encouragement was provided to induce the subject to reach a higher level in each trial. The highest value of force recorded over the three maximum contractions was selected as the reference MVC and used to calculate the sub-maximal force targets. The target forces were expressed as percentages of MVC (\%MVC). After 5 min of rest, the subject trained to control a single motor unit (target unit) from one of the two intramuscular EMG signals. The criteria for this choice were that the action potentials of the target motor unit were clearly distinguishable from other motor units and that the subject was able to maintain a stable discharge rate of approximately 7-10 pulses per second (pps). Following the period of training, the subject was asked to maintain the discharge rate of the target unit at approximately $7-10$ pps for $2 \mathrm{~min}$. This contraction was repeated for as many motor units as the subject could control with visual feedback from the intramuscular EMG signals. Following the contractions performed with feedback on the intramuscular EMG signals, the subject performed a 2-min sustained contraction at 5\% MVC with visual feedback on force. This concluded the experimental session for the vastus medialis 
A

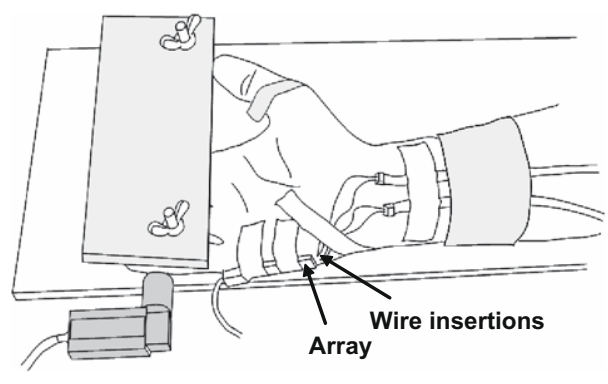

B

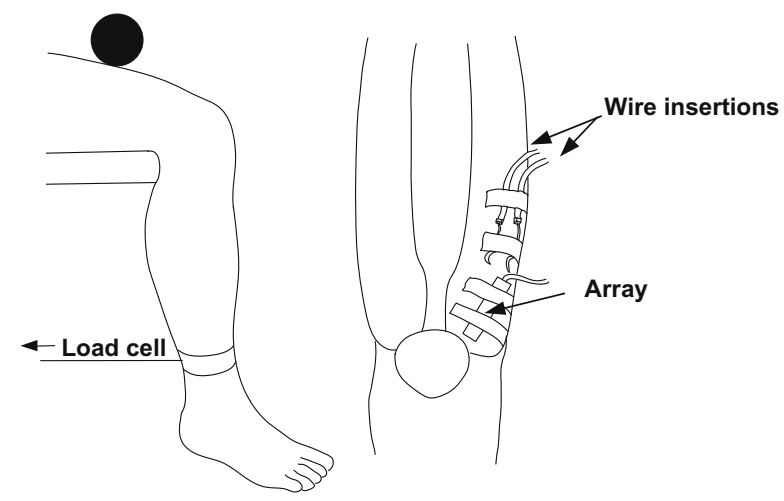

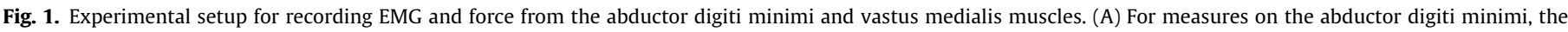

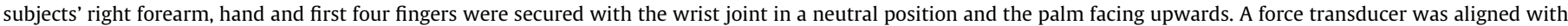

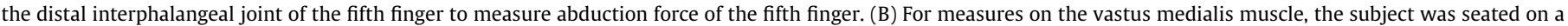
chair with a chain strapped around the ankle which was connected to a load cell (not shown). For both muscles, surface and intramuscular EMG were recorded.

whereas the session on the abductor digiti minimi included additional contractions.

For the abductor digiti minimi, immediately after the 2-min contraction at 5\% MVC, the subject was asked to increase the force from $5 \%$ to $50 \%$ MVC, maintaining the force at this level for $1 \mathrm{~min}$. This additional contraction was performed to investigate the changes in motor unit short-term synchronization and \%DET induced by sustained muscle activity. The force level of 50\% MVC was chosen as a contraction intensity likely sufficient to induce changes in muscle fiber conduction velocity (MFCV) (Merletti et al., 1990) and \%DET (Filligoi and Felici, 1999) but not sufficient to produce substantial shifts of the wire electrodes inside the muscle. Finally, immediately after the sustained contraction, the subject was asked to decrease the force back to 5\% MVC and to maintain this level for a further 2 min.

\subsection{Motor unit short-term synchronization}

Motor unit action potentials were identified from the intramuscular recordings with a decomposition algorithm (McGill et al., 2005) and converted into spike trains. The decomposition of the intramuscular EMG was performed both on the contractions with feedback on the intramuscular EMG and on the contractions with feedback on force at 5\% MVC. Motor unit short-term synchronization was analyzed by generating cross-histograms ( $\pm 100 \mathrm{~ms}$ relative to the reference motor unit discharge, bin width $1 \mathrm{~ms}$ ) of all combinations of motor unit pairs of each 2-min contraction. Cross-histograms with a mean bin count less than 4 were excluded from further analysis (Semmler et al., 1997). The cumulative sum function was used to identify the location of the synchronous peak in the cross-histogram (Ellaway, 1978). Onset of the peak was calculated automatically, by moving in backwards from the mean value of the cumulative sum function until the function had increased again in two bins (not necessarily consecutive). Offset was found with a similar procedure. A significant peak in the cumulative sum function was defined as an increase of more than three standard deviations above the mean of the baseline of the first 50 bins (Davey et al., 1986). The proportion of cross-histograms with a significant peak was calculated.

Synchronization was quantified by CIS (Nordstrom et al., 1992). CIS denotes the number of synchronous discharges in excess of chance, divided by the time during which the motor units in the pair were active. The value of CIS for one 2 -min period was calculated as the mean of all individual values obtained from all available pairs of motor units. Thus, for each 2-min contraction and each motor unit pair, a cross-histogram was computed and CIS calculated. The values for CIS from all the 2-min contractions in each condition were pooled together to get a representative average value of CIS. CIS was preferred over other indexes of motor unit synchronization since it is independent of motor unit discharge rate (Nordstrom et al., 1992).

\subsection{Recurrence quantification analysis}

Surface EMG signals were off-line low-pass filtered with a cutoff frequency of $350 \mathrm{~Hz}$ and high-pass filtered with a cut-off frequency of $10 \mathrm{~Hz}$ (Butterworth filters of order 7). The computation of recurrence maps was performed as described by Eckmann et al. (1987) on non-overlapping epochs of EMG signal of 1-s duration, as previously suggested (Webber et al., 1995; Filligoi and Felici, 1999; Felici et al., 2001). Briefly, the phase-space matrix was constructed by choosing the number of required embedded dimensions. As previously suggested, the number of embedded dimensions was set to 15 (Filligoi and Felici, 1999). The Euclidean distance between the rows of the phase-space matrix was computed to build the distance matrix. The entries of the distance matrix were compared with a threshold to build the binary equivalent of the distance matrix (recurrence plot) (Marwan et al., 2007). The threshold is an important parameter since the number of recurrent points depends on it. In order to compare the results across muscles and subjects, the threshold was computed for each distance matrix so that $10 \%$ of the calculated distances were below the threshold (Eckmann et al., 1987; Marwan et al., 2007). In this way, the number of recurrent points was fixed in all conditions and this allowed comparison between conditions. Diagonal lines of indexes (pixels) with the value 1 in the recurrence plot indicate determinism. The percentage of determinism (\%DET) was calculated as the percentage of pixels with value 1 which formed diagonal lines with respect to the total number of pixels with value 1 . A diagonal line was defined as 2 or more pixels with value 1 in a diagonal. The 120 values of \%DET computed from each 2 -min period were averaged.

The \%DET was computed as described above for both muscles, using the central channel of the array recordings. For the abductor digiti minimi muscle, \%DET was computed using an interelectrode distance of $2.5 \mathrm{~mm}$ as well as $5 \mathrm{~mm}$ for the detection of the surface EMG. Recordings at both interelectrode distances could be obtained from the array (Farina et al., 2003). The computation of \%DET using $5 \mathrm{~mm}$ interelectrode distance was included for comparing the results in the two muscles (vastus medialis EMG was 
detected with $5 \mathrm{~mm}$ interelectrode distance) and for investigating the effect of interelectrode distance on \%DET. It was expected that \%DET would depend on interelectrode distance since the spectral features of the surface EMG are affected by this parameter of the detection system (Farina et al., 2003).

\subsection{Muscle fiber conduction velocity}

Muscle fiber conduction velocity, which is a factor of influence of \%DET (Farina et al., 2002a), was estimated in both muscles in all conditions with a multi-channel algorithm (Farina et al., 2001). The channels used for MFCV estimation were those showing propagation of action potentials, which was determined by visual inspection. MFCV was calculated in epochs of $1 \mathrm{~s}$ (2048 samples), as for $\%$ DET, and the average value over the 2 -min contractions was used for statistical analysis.

\subsection{Statistical analysis}

Values for CIS obtained from the contractions with feedback on intramuscular EMG and with feedback on force were compared with Student paired $t$-tests. In the first experiment, values for CIS, \%DET, and MFCV before and after the 50\% MVC contraction and values for \%DET obtained when using different interelectrode distances (2.5 $\mathrm{mm}$ and $5 \mathrm{~mm}$ ) were also compared with Student paired $t$-tests. Linear regression analysis and multiple linear regression analysis were used to test the relations between the investigated variables (CIS, \%DET, MFCV) and it was applied to the data obtained before and, for the abductor digiti minimi, after the 50\% MVC sustained contraction separately as well as on all data. Values for CIS and \%DET were compared between the two muscles with Student unpaired t-tests. Statistical significance was set at $P<0.05$. Results are presented as mean and standard deviation (SD).

\section{Results}

The results obtained for the abductor digiti minimi muscle were used to investigate the association between \%DET and CIS across subjects within the same muscle before and after the sustained contraction. Moreover, \%DET was computed from the signals recorded from the abductor digiti minimi muscle for interelectrode distances of $2.5 \mathrm{~mm}$ and $5 \mathrm{~mm}$ and the results compared. The results from the vastus medialis were compared with those of the abductor digiti minimi as it was expected that vastus medialis would show substantially lower values of CIS.

Fig. 2 shows an example of cross-histograms obtained for a pair of motor units from abductor digiti minimi during a contraction performed with feedback from intramuscular EMG. The values of CIS obtained from the 2-min contractions with feedback on action potentials were compared with the values obtained from the contractions with feedback on force at 5\% MVC. In the 2-min trials with feedback on motor unit action potentials, 144 motor unit pairs were analyzed; 140 of these pairs showed significant peaks, according to the criteria proposed by Davey et al. (1986), with an average CIS of $0.89 \pm 0.35$. Decomposition of the intramuscular EMG signals from the 2-min contractions at 5\% MVC before the $50 \%$ MVC contraction yielded a total of 184 motor unit pairs; 176 of these pairs had significant peaks and an average CIS of $0.86 \pm 0.27$. A paired $t$-test showed no significant difference between the values of CIS calculated from contractions using either intramuscular or force feedback $(P=0.76)$. Furthermore, the surface EMG amplitude was not different during the two types of contractions (intramuscular and force feedback) (Student's $t$-test; $P=0.62$ ). Comparison of CIS (reported below) computed with feed- back on motor unit action potentials and force in the vastus medialis muscle led to the same conclusion, thus the computation of the degree of motor unit short-term synchronization from contractions with either action potential or force feedback were considered equivalent. The following results report the values of CIS estimated from the 5\% MVC contractions.

In the abductor digiti minimi, after the 50\% MVC contraction, 170 motor unit pairs were extracted from the 5\% MVC contractions; 163 of these pairs showed significant peaks with CIS equal to $0.92 \pm 0.35$. Values of CIS computed before and after the $50 \%$ MVC contraction were positively correlated $\left(R^{2}=0.66, P<0.01\right)$ and significantly different (before $0.86 \pm 0.27$ vs after $0.92 \pm 0.35$; $P<0.01$ ). The values of CIS were computed as an average across $14.8 \pm 7.8$ motor unit pairs per contraction. The SD of CIS across the motor unit pairs (used to obtain the average CIS for each contraction) was ( $n=12$ subjects) $0.38 \pm 0.15$, indicating a rather large variability in CIS depending on the motor unit pair used for the computation.

Fig. 3 shows an example of a recurrence plot obtained from one subject during the $5 \%$ MVC contraction of the abductor digiti minimi muscle performed before the 50\% MVC contraction. The \%DET was calculated from the distance matrix and in this example was 79.4\%. From the group data for the abductor digiti minimi muscle in the $5 \%$ MVC contractions prior to the $50 \%$ MVC contraction, \%DET (computed from the EMG with a $2.5 \mathrm{~mm}$ interelectrode distance) was $81.0 \pm 4.4$ whereas it was $84.2 \pm 3.2$ after the $50 \%$ MVC contraction $\left(R^{2}=0.15, P=0.23\right.$; significantly different $\left.P<0.05\right)$. MFCV decreased from $4.2 \pm 0.8 \mathrm{~m} / \mathrm{s}$ to $3.8 \pm 0.7 \mathrm{~m} / \mathrm{s} \quad\left(R^{2}=0.76\right.$, $P<0.001$; significantly different $P<0.01$ ) after the 50\% MVC contraction with respect to before. Thus, the contraction level chosen was sufficient to induce changes in \%DET and MFCV.

The correlation analysis of CIS and \%DET values for the abductor digiti minimi muscle showed that CIS did not correlate with \%DET. This was observed when the data were pooled together (before and after the 50\% MVC contraction) $\left(R^{2}=0.11, P=0.12\right.$; Fig. 4$)$, or when considering the values before $\left(R^{2}=0.21, P=0.20\right)$ and after $\left(R^{2}=0.18, P=0.15\right)$ the $50 \%$ MVC contraction separately. Moreover, \%DET did not correlate with MFCV (all values: $R^{2}=0.01, P=0.79$; before the contraction: $R^{2}=0.03, P=0.56$; after the contraction: $R^{2}=0.01, P=0.77$ ). Finally, multiple linear regression showed no correlation between the three parameters (CIS, \%DET, and MFCV) in the abductor digiti minimi (all values: $R^{2}=0.22, P=0.50$; before the contraction: $R^{2}=0.44, P=0.59$; after the contraction: $R^{2}=0.18$, $P=0.55$ ).

The relative changes in \%DET after the 50\% MVC contraction with respect to the initial condition $(4.9 \pm 5.7 \%)$ did not correlate with the relative changes in CIS $(0.15 \pm 31.6 \%)\left(R^{2}=0.01\right.$, $P=0.94)$. Moreover, there was no correlation between relative changes in \%DET and MFCV $(-10.3 \pm 8.7 \%)$ induced by the $50 \%$ MVC contraction $\left(R^{2}=0.04, P=0.52\right)$.

From the abductor digiti minimi, the \%DET was calculated in the same conditions as reported above but using double interelectrode distance $(5 \mathrm{~mm})$. The average values before and after the 50\% MVC contraction were in this case $71.6 \pm 5.5 \%$ and $78.9 \pm 4.3 \%$, respectively (significantly different between each other, $P<0.01$ ). These values were both different from the respective values obtained before and after the sustained contraction when \%DET was computed using $2.5 \mathrm{~mm}$ interelectrode distance $(P<0.001)$, which indicated that \%DET depended on the choice of interelectrode distance.

The vastus medialis muscle was analyzed because it was expected that the degree of synchronization would be less in this muscle with respect to the abductor digiti minimi (Kim et al., 2001). Accordingly, out of the 134 motor unit pairs which were detected from the vastus medialis muscle, only 52 pairs showed a significant peak in the cross-histogram. The value of CIS from the pairs that showed significant peaks was $0.08 \pm 0.07$, signifi- 


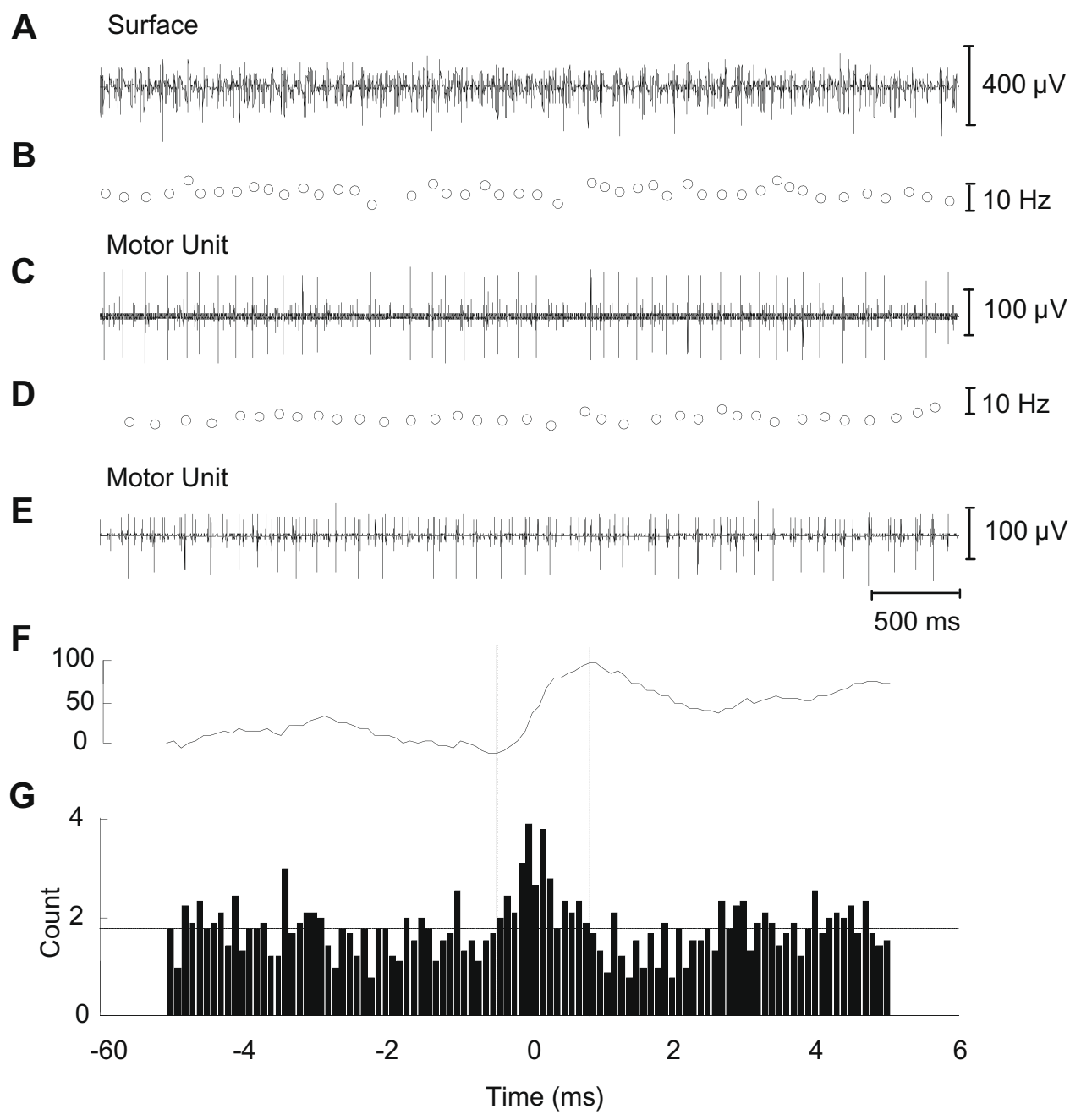

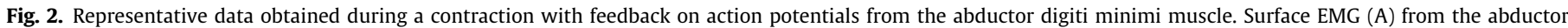

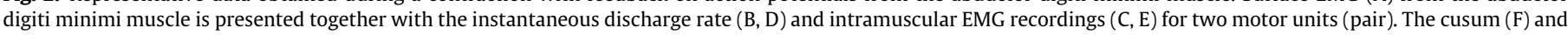

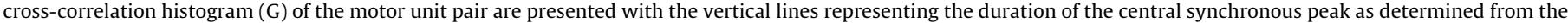
cusum.

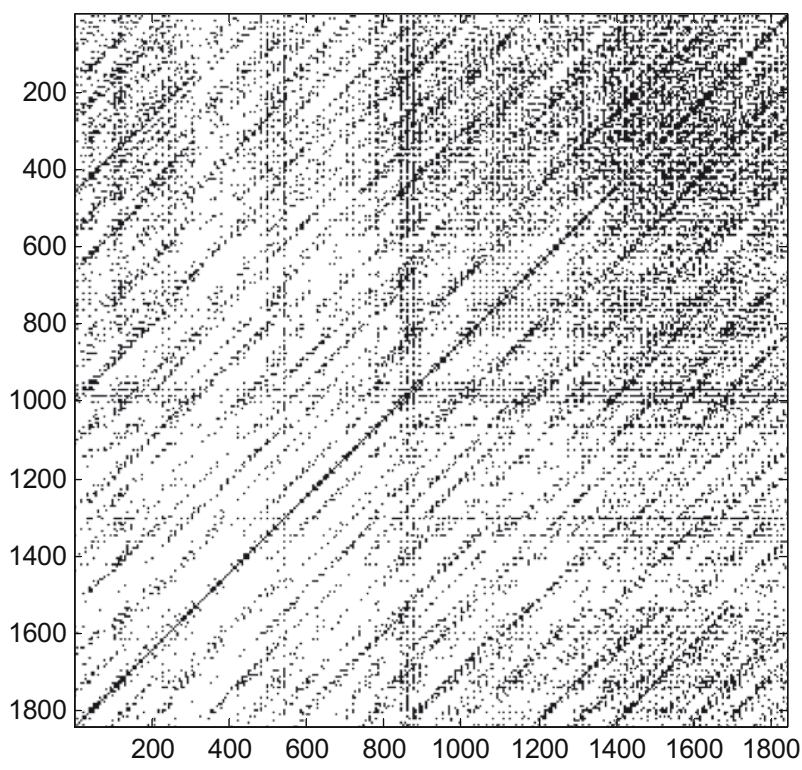

Fig. 3. Representative recurrence plot computed from a contraction at $5 \%$ MVC of the abductor digiti minimi muscle.

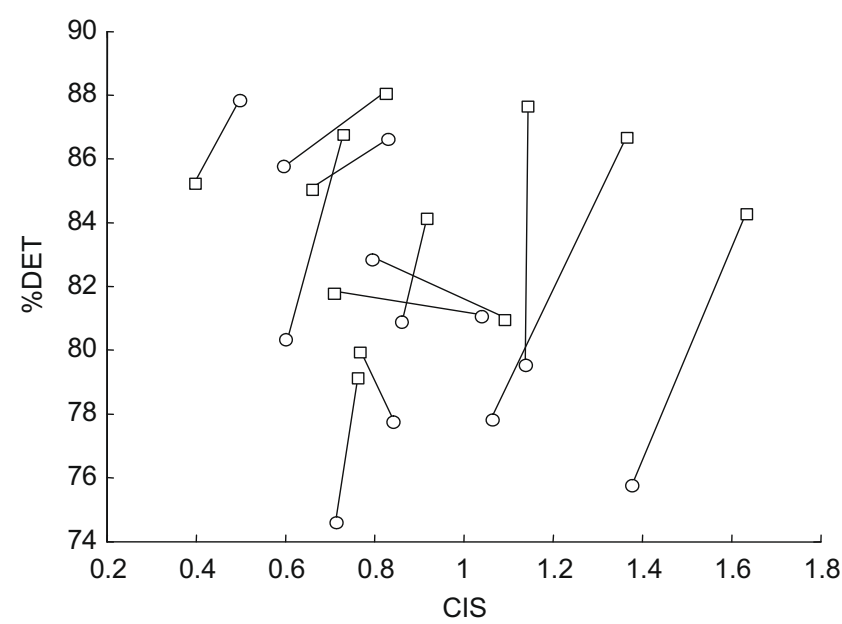

Fig. 4. Scatter plot of the common input strength (CIS) and percent of determinism (\%DET) computed across all data obtained before and after a sustained contraction of the abductor digiti minimi muscle contracting at $50 \%$ of the maximal force $\left(R^{2}=0.11, P=0.12\right)$. The circles correspond to the values before and the squares to the values after the sustained contraction. The lines connect the data for each individual subject. 
cantly lower than the values obtained from the abductor digiti minimi muscle $(P<0.001)$. These results confirmed that the two muscles have substantially different levels of motor unit shortterm synchronization. Despite the very different degrees of synchronization between the two muscles, \%DET estimated from the vastus medialis $(66.9 \pm 8.7 \%)$ was not statistically different $(P=0.12)$ from the values of \%DET estimated from the abductor digiti minimi muscle $(71.6 \pm 5.5 \%)$ when using signals detected with $5 \mathrm{~mm}$ interelectrode distance (the interelectrode distance used for recordings from the vastus medialis). Thus, \%DET could not differentiate the two muscles even though the muscles were at the two extremes of physiological values of CIS observed in human muscles (Datta et al., 1991; De Luca et al., 1993; Kim et al., 2001).

Similar to the results obtained from the abductor digiti minimi muscle, the CIS of the vastus medialis muscle did not correlate with \%DET $\left(R^{2}=0.04, P=0.56\right)$. Moreover, MFCV of the vastus medialis was $3.9 \pm 0.4 \mathrm{~m} / \mathrm{s}$ and did not correlate with \%DET $\left(R^{2}=0.12, P=0.48\right)$. Further, multiple linear regression showed no correlation between the three parameters (CIS, \%DET, and MFCV $)$ in the vastus medialis $\left(R^{2}=0.25, P=0.42\right)$, which was consistent with the results for the abductor digiti minimi.

\section{Discussion}

For the abductor digiti mimimi and vastus medialis muscles, the \%DET extracted from non-linear analysis of the surface EMG did not correlate with a direct measure of motor unit short-term synchronization. Moreover, the values of \%DET computed from the two muscles were not significantly different whereas the direct measure of synchronization showed significantly greater values of synchronization for the abductor digiti minimi muscle. Finally, \%DET depended on the interelectrode distance used for the detection of surface EMG.

The results obtained are at variance with those reported by Del Santo et al. (2006, 2007). The authors showed a correlation between relative changes in \%DET and relative changes in the degree of short-term synchronization (in their study, $R=0.84$ ) (Del Santo et al., 2006) and a correlation between absolute values of \%DET and degree of synchronization during injection of L-acetylcarnitine $(R=0.99)$ (Del Santo et al., 2007). These correlation values could not be reproduced in the current study which was performed without a pharmacological intervention.

The association between CIS and \%DET was investigated in this study in several conditions. First, the linear correlation between CIS and \%DET was analyzed across subjects within each of the two muscles measured. The correlation was not significant in either muscle even though the values of CIS showed a rather large range across subjects (e.g., Fig. 4). Second, the \%DET values were compared between the two muscles. These muscles showed very different degrees of synchronization when assessed with the standard technique based on single unit recordings (CIS, $0.86 \pm 0.27$ vs $0.08 \pm 0.07)$, however the \%DET values computed from the two muscles did not differ $(71.6 \pm 5.5 \%$ vs $66.9 \pm 8.7 \%)$. Third, in the abductor digiti minimi muscle, CIS and \%DET were computed before and after a sustained contraction. Although both measures changed as a consequence of the sustained contraction, their relative changes were not correlated. Overall, these observations indicate that the association between \%DET and the degree of motor unit short-term synchronization is not robust enough to justify the use of recurrence analysis of the surface EMG for investigating motor unit short-term synchronization. The degree of synchronization was estimated from a relatively large number of motor unit pairs, sufficient for an estimate representative of the entire muscle (Semmler and Nordstrom, 1999), thus it is unlikely that the results can be explained by the variability in degree of synchronization when different groups of motor units are investigated. It is likely that \%DET is affected by the degree of motor unit synchronization but not exclusively, thus masking the possibility of obtaining a clear association in practical conditions. This interpretation is partly confirmed by previous simulation analyses (Farina et al., 2002a). However, the low correlation levels between CIS and \%DET may also be due to the large variability among subjects since the correlation analysis was performed across the subject pool. Higher levels of correlation may be obtained when investigating the relation between CIS and \%DET from several measurements on the same subject (Farina et al., 2002a; Del Santo et al., 2006, 2007).

The effect of the interelectrode distance on \%DET values was investigated by comparing the results obtained when using $2.5 \mathrm{~mm}$ or $5 \mathrm{~mm}$ of electrode separation from the same recordings. This comparison could be easily performed with the use of a linear electrode array. The results indicated that values of \%DET obtained with the smaller interelectrode distance $(81.0 \pm 4.4 \%)$ were significantly greater than those obtained from the same detection site but with double the interelectrode distance $(71.6 \pm 5.5 \%)$. In this comparison, the number of recurrence point was fixed to $10 \%$ (as in all other analyses), thus the differences observed cannot be explained by the effect of the threshold value. The dependence of \%DET on the interelectrode distance confirms modeling analyses which showed that \%DET depends on the spectral content of the signal (Farina et al., 2002a), which changes with interelectrode distance (Farina et al., 2002b). The optimal value of interelectrode distance for \%DET computation should be selected on the basis of the sensitivity of the measure, an issue not addressed in this study.

The computation of \%DET implies a choice of several parameters which can substantially affect the results. In this study, the embedding dimension was fixed for both muscles, however it cannot be excluded that a different embedding dimension is optimal for different muscles. Similarly, we adopted the choice of changing the threshold value in order to maintain the number of recurrent points constant across conditions. Although this choice allows comparison across subjects, conditions, and muscles, other choices could have been implemented. A full analysis of the parameters of recurrence quantification analysis was not performed in this study since the focus was on the association with physiological parameters.

In addition to a comparison with \%DET, the present study also reports the analysis of CIS before and after a sustained contraction. The results showed a small, although significant, increase after the sustained contraction, which is in agreement with data shown by Dartnall et al. (2008). However, the results showed large variability across subjects (Fig. 4).

Muscle fiber conduction velocity was analyzed in the present study since it can be a factor affecting \%DET (Farina et al., 2002a). However, since several factors may affect \%DET, it is unlikely that just one factor has a strong linear association to \%DET (Farina et al., 2002a). In accordance, MFCV did not correlate with the values of \%DET in the conditions analyzed. The results obtained for MFCV and CIS before and after the sustained contraction in the abductor digiti minimi further indicated that the changes in \%DET during sustained contractions do not reveal specific changes in the degree of motor unit short-term synchronization or MFCV (Farina et al., 2002a). Rather, \%DET is affected by both these variables and other factors, such as anatomical parameters.

In conclusion, the results of this study indicate that the variable \%DET extracted by non-linear analysis of the surface EMG cannot be used as a measure of the degree of motor unit short-term synchronization or of the relative changes in motor unit short-term synchronization during sustained contractions. The degree of motor unit synchronization, which is a clinically relevant measure of motor unit behavior, should thus be esti- 
mated directly from the cross-histograms of single motor unit spike trains.

\section{Acknowledgements}

The authors are grateful to Dr. Annie Schmied for reading and commenting on the final version of the manuscript.

The study was partly supported by the European Project TREMOR (Contract \#224051) (D.F.).

\section{References}

Buchthal F, Madsen A. Synchronous activity in normal and atrophic muscle. Electroencephalogr Clin Neurophysiol 1950;2:425-44.

Dartnall TJ, Nordstrom MA, Semmler JG. Motor unit synchronization is increased in biceps brachii after exercise-induced damage to elbow flexor muscles. J Neurophysiol 2008;99:1008-19.

Datta AK, Farmer SF, Stephens JA. Central nervous system pathways underlying synchronization of human motor unit firing studies during voluntary contractions. J Physiol 1991;432:401-25.

Davey NJ, Ellaway PH, Stein RB. Statistical limits for detecting change in the cumulative sum derivative of the peristimulus time histogram. J Neurosci Methods 1986;17:153-66.

Del Santo F, Gelli F, Schmied A, Vedel JP, Rossi A, Mazzocchio R. Motor unit synchronous firing as revealed by determinism of surface myoelectric signal. J Neurosci Methods 2006;155:116-21.

Del Santo F, Gelli F, Mazzocchio R, Rossi A. Recurrence quantification analysis of surface EMG detects changes in motor unit synchronization induced by recurrent inhibition. Exp Brain Res 2007;178:308-15.

De Luca CJ, Roy AM, Erim Z. Synchronization of motor-unit firings in several human muscles. J Neurophysiol 1993;70:2010-23.

Eckmann JP, Kamphorst SO, Ruelle D. Recurrence plots of dynamical systems. Europhys Lett 1987;4:973-7.

Ellaway P. Cumulative sum technique and its application to the analysis of peristimulus time histograms. Electroencephalogr Clin Neurophysiol 1978;45:302-4.

Farina D, Muhammad W, Fortunato E, Meste O, Merletti R, Rix H. Estimation of single motor unit conduction velocity from surface electromyogram signals detected with linear electrode arrays. Med Biol Eng Comput 2001;39:225-36.

Farina D, Fattorini L, Felici F, Filligoi G. Nonlinear surface EMG analysis to detect changes of motor unit conduction velocity and synchronization. J Appl Physiol 2002a;93:1753-63.
Farina D, Cescon C, Merletti R. Influence of anatomical, physical, and detectionsystem parameters on surface EMG. Biol Cybern 2002b;86:445-56.

Farina D, Gazzoni M, Merletti R. Assessment of low back muscle fatigue by surface EMG signal analysis: methodological aspects. J Electromyogr Kinesiol 2003;13:319-32.

Farina D, Merletti R, Enoka RM. The extraction of neural strategies from the surface EMG. J Appl Physiol 2004;96:1486-95.

Farmer SF, Bremner FD, Halliday DM, Rosenberg JR, Stephens JA. The frequency content of common synaptic inputs to motoneurones studied during voluntary isometric contraction in man. J Physiol 1993;470:127-55.

Felici F, Rosponi A, Sbriccoli P, Filligoi GC, Fattorini L, Marchetti M. Linear and nonlinear analysis of surface electromyograms in weightlifters. Eur J Appl Physiol 2001;84:337-42.

Filligoi G, Felici F. Detection of hidden rhythms in surface EMG signals with a nonlinear time-series tool. Med Eng Phys 1999;21:439-48.

Kim MS, Masakado Y, Tomita Y, Chino N, Pae YS, Lee KE. Synchronization of single motor units during voluntary contractions in the upper and lower extremities. Clin Neurophysiol 2001;112:1243-9.

Marwan N, Romano MC, Thiel M, Kurths J. Recurrence plots for the analysis of complex systems. Phys Rep 2007;438:237-329.

McGill KC, Lateva ZC, Marateb HR. EMGLAB: an interactive EMG decomposition program. J Neurosci Methods 2005;149:121-33.

Merletti R, Knaflitz M, De Luca CJ. Myoelectric manifestations of fatigue in voluntary and electrically elicited contractions. J Appl Physiol 1990;69:1810-20.

Milner-Brown HS, Stein RB, Yemm R. The contractile properties of human motor units during voluntary isometric contractions. J Physiol 1973;228:285-306.

Milner-Brown HS, Stein RB, Lee RG. Synchronization of human motor units: possible roles of exercise and supraspinal reflexes. Electroencephalogr Clin Neurophysiol 1975;38:245-54.

Nordstrom MA, Fuglevand AJ, Enoka RM. Estimating the strength of common input to human motoneurons from the cross-correlogram. J Physiol 1992;453:547-74.

Sears TA, Stagg D. Short-term synchronization of intercostal motoneurone activity. J Physiol 1976;263:357-81.

Semmler JG, Nordstrom MA, Wallance CJ. Relationship between motor unit shortterm synchronization and common drive in human first dorsal interosseous muscle. Brain Res 1997;767:314-20.

Semmler JG, Nordstrom MA. A comparison of cross-correlation and surface EMG techniques used to quantify motor unit synchronization in humans. J Neurosc Methods 1999:90:47-55.

Semmler JG. Motor unit synchronization and neuromuscular performance. Exerc Sport Sci Rev 2002;30:8-14.

Webber Jr CL, Schmidt MA, Walsh JM. Influence of isometric loading on biceps EMC dynamics as assessed by linear and nonlinear tools. J Appl Physio 1995;78:814-22. 